the frequency of miniature potentials, and the delayed potentiation of the endplate potential after a tetanus in high magnesium solutions. Other factors, notably depletion of available transmitter, are obviously also concerned in determining the amount of trans. mitter released. Mobilization of transmitter by preceding impulses remains an important, indeed probably the basic factor, in post-activation potentiation.

Department of Physiology, Australian National University, Canberra.

${ }^{1}$ Eccles, J. C., 'The Physiology of Nerve Cells', 211 (Oxford Univ. Press, 1957).

${ }^{2}$ Liley, A. W., J. Physiol., 132, 650 (1956)

${ }^{3}$ Liley, A, W., and North, K., J. Neurophysiol., 16, 509 (1953).

' Liley, A. W., J. Physiol., 133, 571 (1956).

\section{Oxygen Uptake of Glycerol-extracted Muscle Fibres}

IN the ten years that have passed since Szent. Györgyi ${ }^{1}$ introduced the use of the glycerol-extracted muscle fibre, it has proved extremely useful for investigating the molecular events underlying muscular contraction. Usually, the fibre has been regarded as a 'contractile skeleton' ; on this basis its response to a chemical agent has commonly been interpreted as a direct response of the contractile elements, rather than an effect mediated or influenced by other still functional components of the muscle cell.

When exploratory studies of the activities of adenosine triphosphatase (Wilson, J. A., and Guthe, K. F., unpublished work) suggested that glycerol fibres take up inorganic phosphate under certain conditions, the question arose as to the possible persistence of a broad range of enzymes and enzyme systems in the fibre. Although Perry ${ }^{2}$ has directed attention to the desirability of investigating this matter, little information is at present available on the properties of the fibre apart from the contractile systern itself and one or more relaxing factors ${ }^{3}$. As a preliminary approach to the question, we have sought in the present study to determine the respiratory capacities of glycerol-extracted rabbit psoas fibres.

Freshly removed fibres were stored in 50 per cent glycerol $(\mathrm{v} / \mathrm{v})$ at $-17^{\circ} \mathrm{C}$. for periods of four weeks to four months. As desired, they were removed from the

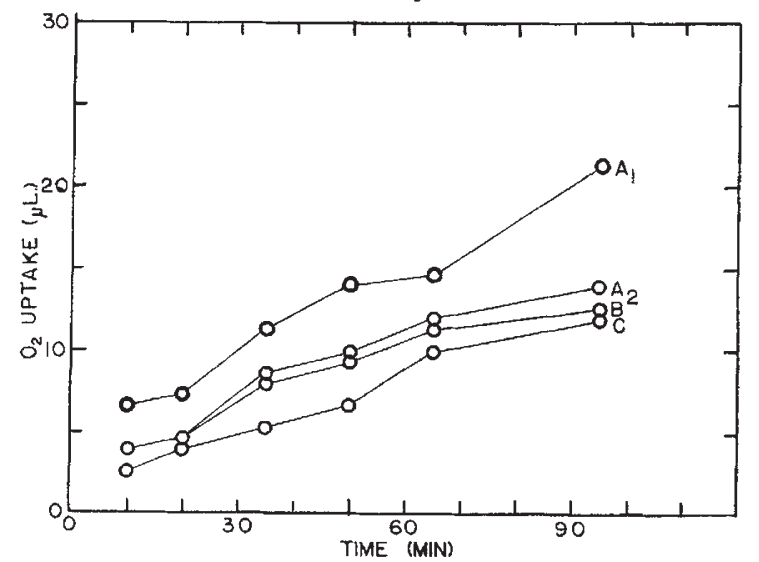

Fig. 1. Oxygen consumption of rabbit psoas fibre homogenates at $37^{\circ} \mathrm{C}$. In all experiments, a total flask volume of $1.8 \mathrm{ml}$. was used containing $0.045 \mathrm{gm}$. tissue. Final concentrations were as follows : sucrose, $0.21 M$, and phosphate, $0.017 M(p \mathbf{H} 7 \cdot 0)$. Curve $A_{x}$ fresh flbre without succinate; curve $A_{3}$, fresh fibre in $0.0017{ }_{l}$ succinate; curve $B, 5$-weeks glycerol-extracted fibre in $0.0017 M$ succinate; curve $C$, 4-months glycerol-extracted fibre in $0.0017 M$ glycerol, rinsed briefly in $0.25 M$ sucrose, and homogenized in 9-30 volumes of fresh $0.25 M$ sucrose. Manometric measurements of the oxygen consumption of such homogenates are illustrated in Fig. 1. Curve $A_{3}$ shows the endogenous oxygen uptake of a homogenate of fresh fibres prior to storage in glycerol, and curve $A_{2}$ the slightly lower rate exhibited by the same homogenate in the presence of $0.0017 M$ succinate. In curves $B$ and $C$ are illustrated the oxygen consumptions of other homogenates, prepared from fibres stored in glycerol for five weeks and four months, respectively. Of particular interest is the fact that the homogenates of glycerol fibres consume oxygen at rates substantially similar to those shown by homogenates of fresh fibres.

These results have been confirmed by additional experiments, which have shown also that homogenates of fibres stored six weeks in glycerol consume oxygen as rapidly without added substrate with succinate.

The above findings imply that a functional respiratory enzyme system is retained in the glycerol fibres. Support for this view was obtained from spectroscopic observations, which revealed that an intact cytochrome system persists in the glycerol fibres even after four months. Although the glycerol fibres, like fresh fibres, contain relatively low concentrations of cytochromes, the absorption bands of components $a+a_{3}, b$, and $c$ were in all cases readily detectable in the presence of sodium dithionite, and particularly after intensification of the absorption spectra at the temperature of liquid nitrogen. The relative band intensities in fresh fibres were observed to be $b=c>a+a_{3}$; those in the glycerol fibres $b>c>a+a_{3}$, suggesting that a small part of the cytochrome $c$ may be extracted from the fibres during the period of storage at low temperature.

Further evidence for the integrity of the cytochrome system after storage in glycerol was provided by comparative studies on rabbit liver mitochondria, isolated in $0.25 M$ sucrose. The mitochondria, after storage for six weeks in 50 per cent glycerol at $-17^{\circ} \mathrm{C}$., consumed oxygen at a rate about ten times that of the muscle homogenates. This oxygen uptake was almost entirely abolished in $0.001 M$ cyanide.

These findings indicate that the glycerol-extracted muscle fibre, as customarily prepared for physiological studies, contains respiratory substrate and the means for oxidizing it. It is perhaps not surprising that the fibre respires, in view of the marked vitality of many types of animal cells after low-temperature storage in glycerol media. Clearly, a fibre that not only contracts but also respires may well retain other significant biochemical activities. The latter unquestionably merit attention in assessing the contractile properties of the fibre. In particular, the status of the mitochondrial oxidative phosphorylation mechanism becomes an important point; if it remains intact, the products of phosphorylation and transphosphorylation could themselves exert potent effects on the contractile mechanism, as well as influence its response to a wide range of physiologically active substances.

This work was supported in part by a grant from the United States Public Health Service (RG-5903).

\section{James A. Wilson Paul R. Elliott* \\ Karl F. GUthe David G. Shappirio}

Dept. of Zoology, University of Michigan.

* Predoctoral Fellow, U.S. Public Health Service ( $C F-9138)$.

2 Szent-Györgyi, A., Biol. Bull., 96, 140 (1949).

3 Molnar, J., and Lorand, L, Nature, 183, 1032 (1959). Briggs, F. N. 